\title{
Fuzzy $r$-minimal Continuous Functions Between Fuzzy Minimal Spaces and Fuzzy Topological Spaces
}

\author{
Won Keun Min \\ Department of Mathematics, Kangwon National University, Chuncheon, 200-701, Korea
}

\begin{abstract}
In this paper, we introduce the concepts of fuzzy $r$-minimal continuous function and fuzzy $r$-minimal open function between a fuzzy $r$-minimal space and a fuzzy topological space. We also investigate characterizations and properties for such functions.
\end{abstract}

Key Words : $r$-minimal structure, fuzzy $r$-minimal continuous, fuzzy $r$-minimal open function

\section{Introduction}

The concept of fuzzy set was introduced by Zadeh [10]. Chang [2] defined fuzzy topological spaces using fuzzy sets. In $[3,8]$, Chattopadhyay, Hazra and Samanta introduced a smooth topological space which is a generalization of fuzzy topological space.

In [9], Yoo et al. introduced the concept of fuzzy $r$ minimal space which is an extension of the smooth topological space. The concept of fuzzy $r-M$ continuity was also introduced and investigated in [9]. In this paper, we introduce the concepts of fuzzy $r$-minimal continuous function and fuzzy $r$-minimal open function on fuzzy $r$ minimal spaces and investigate characterizations for such functions.

\section{Preliminaries}

Let $I$ be the unit interval $[0,1]$ of the real line. A member $A$ of $I^{X}$ is called a fuzzy set of $X$. By $\tilde{\mathbf{0}}$ and $\tilde{\mathbf{1}}$ we denote constant maps on $X$ with value 0 and 1 , respectively. For any $A \in I^{X}, A^{c}$ denotes the complement $\tilde{\mathbf{1}}-\mathbf{A}$. All other notations are standard notations of fuzzy set theory.

An fuzzy point $x_{\alpha}$ in $X$ is a fuzzy set $x_{\alpha}$ defined as follows

$$
x_{\alpha}(y)= \begin{cases}\alpha, & \text { if } y=x \\ 0, & \text { if } y \neq x .\end{cases}
$$

A fuzzy point $x_{\alpha}$ is said to belong to a fuzzy set $A$ in $X$, denoted by $x_{\alpha} \in A$, if $\alpha \leq A(x)$ for $x \in X$. A fuzzy set $A$ in $X$ is the union of all fuzzy points which belong to $A$.

Let $f: X \rightarrow Y$ be a function and $A \in I^{X}$ and $B \in I^{Y}$.

Manuscript received Oct. 5, 2009; revised Oct. 22, 2009;

Accepted Dec. 5, 2009.

Corresponding Author : wkmin@kangwon.ac.kr (Won Keun Min)
Then $f(A)$ is a fuzzy set in $Y$, defined by

$$
f(A)(y)= \begin{cases}\sup _{z \in f^{-1}(y)} A(z), & \text { if } f^{-1}(y) \neq \emptyset, \\ 0, & \text { otherwise }\end{cases}
$$

for $y \in Y$ and $f^{-1}(B)$ is a fuzzy set in $X$, defined by $f^{-1}(B)(x)=B(f(x)), x \in X$.

A fuzzy topology (or smooth topology) [3,5] on $X$ is a map $\mathcal{T}: I^{X} \rightarrow I$ which satisfies the following properties:

(1) $\mathcal{T}(\tilde{0})=\mathcal{T}(\tilde{1})=1$.

(2) $\mathcal{T}\left(A_{1} \wedge A_{2}\right) \geq \mathcal{T}\left(A_{1}\right) \wedge \mathcal{T}\left(A_{2}\right)$ for $A_{1}, A_{2} \in I^{X}$.

(3) $\mathcal{T}\left(\bigvee A_{i}\right) \geq \bigwedge \mathcal{T}\left(A_{i}\right)$ for $A_{i} \in I^{X}$.

The pair $(X, \mathcal{T})$ is called a fuzzy topological space. And $A \in I^{X}$ is said to be fuzzy r-open (resp., fuzzy $r$ closed) if $\mathcal{T}(A) \geq r$ (resp., $\mathcal{T}\left(A^{c}\right) \geq r$ ).

The $r$-closure of $A$, denoted by $\operatorname{cl}(A, r)$, is defined as $\operatorname{cl}(A, r)=\cap\left\{B \in I^{X}: A \subseteq B\right.$ and $B$ is fuzzy $r$-closed $\}$.

The $r$-interior of $A$, denoted by $\operatorname{int}(A, r)$, is defined as $\operatorname{int}(A, r)=\cup\left\{B \in I^{X}: B \subseteq A\right.$ and $B$ is fuzzy $r$-open $\}$.

Definition 2.1 ([9]). Let $X$ be a nonempty set and $r \in$ $(0,1]=I_{0}$. A fuzzy family $\mathcal{M}: I^{X} \rightarrow I$ on $X$ is said to have a fuzzy $r$-minimal structure if the family

$$
\mathcal{M}_{r}=\left\{A \in I^{X} \mid \mathcal{M}(A) \geq r\right\}
$$

contains $\tilde{\mathbf{0}}$ and $\tilde{\mathbf{1}}$.

Then the $(X, \mathcal{M})$ is called a fuzzy r-minimal space (simply $r$-FMS) if $\mathcal{M}$ has a fuzzy $r$-minimal structure. Every member of $\mathcal{M}_{r}$ is called a fuzzy r-minimal open set. A fuzzy set $A$ is called a fuzzy r-minimal closed set if the complement of $A$ (simply, $A^{c}$ ) is a fuzzy $r$-minimal open set.

Let $(X, \mathcal{M})$ be an $r$-FMS and $r \in I_{0}$. The fuzzy $r$ minimal closure and the fuzzy $r$-minimal interior of $A$ [9], 
denoted by $m C(A, r)$ and $m I(A, r)$, respectively, are defined as

$$
\begin{gathered}
m C(A, r)=\cap\left\{B \in I^{X}: B^{c} \in \mathcal{M}_{r} \text { and } A \subseteq B\right\} \\
m I(A, r)=\cup\left\{B \in I^{X}: B \in \mathcal{M}_{r} \text { and } B \subseteq A\right\} .
\end{gathered}
$$

Theorem 2.2 ([9]). Let $(X, \mathcal{M})$ be an $r$-FMS and $A, B$ in $I^{X}$.

(1) $m I(A, r) \subseteq A$ and if $A$ is a fuzzy $r$-minimal open set, then $m I(A, r)=A$.

(2) $A \subseteq m C(A, r)$ and if $A$ is a fuzzy $r$-minimal closed set, then $m C(A, r)=A$.

(3) If $A \subseteq B$, then $m I(A, r) \subseteq m I(B, r)$ and $m C(A, r) \subseteq m C(B, r)$.

(4) $m I(A, r) \cap m I(B, r) \supseteq m I(A \cap B, r)$ and $m C(A, r) \cup m C(B, r) \subseteq m C(A \cup B, r)$.

(5) $m I(m I(A, r), r)=m I(A, r)$ and $m C(m C(A, r), r)=m C(A, r)$.

(6) $\tilde{\mathbf{1}}-m C(A, r)=m I(\tilde{\mathbf{1}}-A, r)$ and $\tilde{\mathbf{1}}-m I(A, r)=$ $m C(\tilde{\mathbf{1}}-A, r)$.

Definition 2.3 ([9]). Let $\left(X, \mathcal{M}_{X}\right)$ and $\left(Y, \mathcal{M}_{Y}\right)$ be two $r$-FMS's. Then a function $f: X \rightarrow Y$ is said to be

(1) fuzzy $r$-M continuous if for every fuzzy $r$-minimal open set $A$ in $Y, f^{-1}(A)$ is fuzzy $r$-minimal open in $X$,

(2) fuzzy $r$-M open if for every fuzzy $r$-minimal open set $G$ in $X, f(G)$ is fuzzy $r$-minimal open in $Y$.

\section{Fuzzy $r$-minimal continuous function and fuzzy $r$-minimal open functions}

Definition 3.1. Let $\left(X, \mathcal{M}_{X}\right)$ be an $r$-FMS and $(Y, \sigma)$ a fuzzy topological space. Then $f: X \rightarrow Y$ is said to be fuzzy $r$-minimal continuous if for every fuzzy $r$-open set $A$ in $Y, f^{-1}(A)$ is fuzzy $r$-minimal open in $X$.

Theorem 3.2. Let $f: X \rightarrow Y$ be a function between an $r$ FMS $\left(X, \mathcal{M}_{X}\right)$ and a fuzzy topological space $(Y, \sigma)$. Then we have the following:

(1) $f$ is fuzzy $r$-minimal continuous.

(2) $f^{-1}(B)$ is a fuzzy $r$-minimal closed set for each fuzzy $r$-closed set $B$ in $Y$.

(3) $f(m C(A, r)) \subseteq \operatorname{cl}(f(A), r)$ for $A \in I^{X}$.

(4) $m C\left(f^{-1}(B), r\right) \subseteq f^{-1}(c l(B, r))$ for $B \in I^{Y}$.

(5) $f^{-1}(\operatorname{int}(B, r)) \subseteq m I\left(f^{-1}(B), r\right)$ for $B \in I^{Y}$.

Then $(1) \Leftrightarrow(2) \Rightarrow(3) \Leftrightarrow(4) \Leftrightarrow(5)$.

Proof. (1) $\Leftrightarrow(2)$ Obvious.

$(2) \Rightarrow(3)$ For $A \in I^{X}$,

$f^{-1}(\operatorname{cl}(f(A), r))$

$=f^{-1}\left(\cap\left\{F \in I^{Y}: f(A) \subseteq F, F\right.\right.$ is fuzzy $r$-closed $\left.\}\right)$
$=\cap\left\{f^{-1}(F): A \subseteq f^{-1}(F), F\right.$ is fuzzy $r$-closed $\}$

$\supseteq \cap\left\{K \in I^{X}: A \subseteq K, K\right.$ is fuzzy $r$-minimal closed $\}$ $=m C(A, r)$.

Hence $f(m C(A, r)) \subseteq c l(f(A), r)$.

$(3) \Leftrightarrow(4)$ Fort $B \in I^{Y}$, from (3), it follows

$f\left(m C\left(f^{-1}(B), r\right)\right) \subseteq \operatorname{cl}\left(f\left(f^{-1}(B)\right), r\right) \subseteq c l(B, r)$.

Hence (4) is obtained and similarly, we get $(4) \Rightarrow(3)$.

$(4) \Leftrightarrow(5)$ From Theorem 2.2, it is obvious.

Example 3.3. Let $X=I$ and let us consider two fuzzy sets $A, B$ defined as

$$
\begin{gathered}
A(x)=\frac{1}{2} x, \quad x \in X ; \\
B(x)=-\frac{1}{2}(x-1), \quad x \in X .
\end{gathered}
$$

Consider a fuzzy family

$$
\mathcal{M}(U)= \begin{cases}\frac{1}{2}, & \text { if } U=\tilde{\mathbf{0}}, \tilde{\mathbf{1}} \\ \frac{2}{3}, & \text { if } U=A, B \\ 0, & \text { otherwise }\end{cases}
$$

and a fuzzy topology

$$
\sigma(U)= \begin{cases}1, & \text { if } U=\tilde{\mathbf{0}}, \tilde{\mathbf{1}}, \mathbf{A}, \mathbf{B} \\ \frac{1}{3}, & \text { if } U=A \cap B, A \cup B \\ 0, & \text { otherwise. }\end{cases}
$$

Then the identity function $f:(X, \mathcal{M}) \rightarrow(X, \sigma)$ satisfies the part (3) in Theorem 3.2 but $f$ is not fuzzy $\frac{1}{3}$-minimal continuous.

Let $X$ be a nonempty set and $\mathcal{M}: I^{X} \rightarrow I$ a fuzzy family on $X$. The fuzzy family $\mathcal{M}$ is said to have the property $(\mathcal{U})[9]$ if for $A_{i} \in \mathcal{M}(i \in J)$,

$$
\mathcal{M}\left(\cup A_{i}\right) \geq \wedge \mathcal{M}\left(A_{i}\right) .
$$

Theorem 3.4. Let $f: X \rightarrow Y$ be a function between an $r$-FMS $\left(X, \mathcal{M}_{X}\right)$ and a fuzzy topological space $(Y, \sigma)$. If $\mathcal{M}_{X}$ has the property $(\mathcal{U})$, then $f$ is fuzzy $r$-minimal continuous iff for a fuzzy point $x_{\alpha}$ in $X$ and each fuzzy $r$-open set $V$ containing $f\left(x_{\alpha}\right)$, there is a fuzzy $r$-minimal open set $U$ containing $x_{\alpha}$ such that $f(U) \subseteq V$.

Proof. Let $f$ be fuzzy $r$-minimal continuous, then for fuzzy point $x_{\alpha}$ in $X$ and each fuzzy $r$-open set $V$ containing $f\left(x_{\alpha}\right)$, from Theorem $3.2(5), x_{\alpha} \in f^{-1}(V)=$ $f^{-1}(\operatorname{int}(V, r)) \subseteq m I\left(f^{-1}(V), r\right)$. So there exists a fuzzy $r$-minimal open set $U$ containing $x_{\alpha}$ such that $x_{\alpha} \in U \subseteq$ $f^{-1}(V)$. Hence the result is obtained.

For the converse, let a fuzzy set $A$ be fuzzy $r$-open in $Y$. Then by hypothesis, we have $\cup U=f^{-1}(A)$ for each fuzzy $r$-minimal open set $U$ in $X$. Hence by the property $(\mathcal{U}), f^{-1}(A)$ is fuzzy $r$-minimal open. 
Corollary 3.5. Let $f: X \rightarrow Y$ be a function between an $r$-FMS $\left(X, \mathcal{M}_{X}\right)$ and a fuzzy topological space $(Y, \tau)$. If $\mathcal{M}_{X}$ has property $(\mathcal{U})$, then the following are equivalent:

(1) $f$ is fuzzy $r$-minimal continuous.

(2) For fuzzy point $x_{\alpha}$ in $X$ and each fuzzy $r$-open set $V$ containing $f\left(x_{\alpha}\right)$, there is a fuzzy $r$-minimal open set $U$ containing $x_{\alpha}$ such that $f(U) \subseteq V$.

(3) $f^{-1}(B)$ is fuzzy $r$-minimal closed, for each fuzzy $r$-closed set $B$ in $Y$.

(4) $f(m C(A, r)) \subseteq c l(f(A), r)$ for $A \in I^{X}$.

(5) $m C\left(f^{-1}(B), r\right) \subseteq f^{-1}(c l(B, r))$ for $B \in I^{Y}$.

(6) $f^{-1}(\operatorname{int}(B, r)) \subseteq m I\left(f^{-1}(B), r\right)$ for $B \in I^{Y}$.

Proof. It follows from Theorem 3.2 and Theorem 3.4.

Definition 3.6. Let $(X, \sigma)$ be a fuzzy topological space and $\left(Y, \mathcal{M}_{Y}\right)$ an $r$-FMS. Then $f:(X, \sigma) \rightarrow\left(Y, \mathcal{M}_{Y}\right)$ is said to be fuzzy $r$-minimal open if for every fuzzy $r$-open set $A$ in $X, f(A)$ is fuzzy $r$-minimal open in $Y$.

Theorem 3.7. Let $f: X \rightarrow Y$ be a function on a fuzzy topological space $(X, \sigma)$ and an $r$-FMS $\left(Y, \mathcal{M}_{Y}\right)$.

(1) $f$ is fuzzy $r$-minimal open.

(2) $f(\operatorname{int}(A, r)) \subseteq m I(f(A), r)$ for $A \in I^{X}$.

(3) $\operatorname{int}\left(f^{-1}(B), r\right) \subseteq f^{-1}(m I(B), r)$ for $B \in I^{Y}$.

Then $(1) \Rightarrow(2) \Leftrightarrow(3)$.

Proof. $(1) \Rightarrow(2)$ For $A \in I^{X}$,

$f(\operatorname{int}(A), r)$

$=f\left(\cup\left\{B \in I^{X}: B \subseteq A, B\right.\right.$ is fuzzy $r$-open $\left.\}\right)$

$=\bigcup\left\{f(B) \in I^{Y}: f(B) \subseteq f(A)\right.$,

$f(B)$ is fuzzy $r$-minimal open

$\subseteq \cup\left\{U \in I^{Y}: U \subseteq f(A), U\right.$ is fuzzy $r$-minimal open $\}$ $=m I(f(A), r)$.

Hence $f(\operatorname{int}(A), r) \subseteq m I(f(A), r)$.

(2) $\Leftrightarrow(3)$ For $B \in I^{Y}$, from (3) it follows that

$f\left(\operatorname{int}\left(f^{-1}(B), r\right)\right) \subseteq m I\left(f\left(f^{-1}(B)\right), r\right) \subseteq m I(B, r)$.

This implies (3). Similarly, we get the implication $(3) \Rightarrow$ (2).

Remark 3.8. In Example 3.3, consider the identity function $f:(X, \sigma) \rightarrow\left(X, \mathcal{M}_{X}\right)$. Then $f$ satisfies the statement (2) in Theorem 3.7, but it is not fuzzy $r$-minimal open.

Lemma 3.9. Let $f: X \rightarrow Y$ be a function on a fuzzy topological space $(X, \sigma)$ and an $r$-FMS $\left(Y, \mathcal{M}_{Y}\right)$. If $f$ is fuzzy $r$-minimal open, then $f(A)=m I(f(A), r)$ for every fuzzy $r$-open set $A$ in $X$.

Proof. It follows from Theorem 2.2 (1).

Theorem 3.10. ([9]) Let $(X, \mathcal{M})$ be an $r$-FMS with the property $(\mathcal{U})$. Then

(1) $m I(A, r)=A$ if and only if $A \in \mathcal{M}_{r}$ for $A \in I^{X}$.

(2) $m C(A, r)=A$ if and only if $A^{c} \in \mathcal{M}_{r}$ for $A \in I^{X}$
From Lemma 3.9 and Theorem 3.10, obviously the next corollary is obtained:

Corollary 3.11. Let $f:(X, \sigma) \rightarrow\left(Y, \mathcal{M}_{Y}\right)$ be a function on a fuzzy topological space $(X, \sigma)$ and an $r$-FMS $\left(Y, \mathcal{M}_{Y}\right)$. If $\mathcal{M}_{Y}$ has property $(\mathcal{U})$, then the following are equivalent:

(1) $f$ is fuzzy $r$-minimal open.

(2) $f(\operatorname{int}(A, r)) \subseteq m I(f(A), r)$ for $A \in I^{X}$.

(3) $\operatorname{int}\left(f^{-1}(B), r\right) \subseteq f^{-1}(m I(B, r))$ for $B \in I^{Y}$.

Definition 3.12. Let $(X, \sigma)$ be a fuzzy topological space and $\left(Y, \mathcal{M}_{Y}\right)$ be an $r$-FMS. Then $f:(X, \sigma) \rightarrow\left(Y, \mathcal{M}_{Y}\right)$ is said to be fuzzy $r$-minimal closed if for every fuzzy $r$ minimal closed set $A$ in $X, f(A)$ is fuzzy $r$-minimal closed in $Y$.

Theorem 3.13. Let $f:(X, \sigma) \rightarrow\left(Y, \mathcal{M}_{Y}\right)$ be a function on a fuzzy topological space $(X, \sigma)$ and an $r$-FMS $\left(Y, \mathcal{M}_{Y}\right)$.

(1) $f$ is fuzzy $r$-minimal closed.

(2) $m C(f(A), r) \subseteq f(c l(A, r))$ for $A \in I^{X}$.

(3) $f^{-1}(m C(B, r)) \subseteq c l\left(f^{-1}(B), r\right)$ for $B \in I^{Y}$.

Then $(1) \Rightarrow(2) \Leftrightarrow(3)$.

Proof. It is similar to the proof of Theorem 3.7.

Lemma 3.14. Let $f:(X, \sigma) \rightarrow\left(Y, \mathcal{M}_{Y}\right)$ be a function on a fuzzy topological space $(X, \sigma)$ and an $r$-FMS $\left(Y, \mathcal{M}_{Y}\right)$. If $f$ is fuzzy $r$-minimal closed, then $f(A)=m C(f(A), r)$ for every fuzzy $r$-minimal closed set $A$ in $X$.

Proof. It is obvious.

Corollary 3.15. Let $f: X \rightarrow Y$ be a function on a fuzzy topological space $(X, \sigma)$ and an $r$-FMS $\left(Y, \mathcal{M}_{Y}\right)$. If $\mathcal{M}_{Y}$ has property $(\mathcal{U})$, then the following are equivalent:

(1) $f$ is fuzzy $r$-minimal closed.

(2) $m C(f(A), r) \subseteq f(c l(A), r)$ for $A \in I^{X}$.

(3) $f^{-1}(m C(B, r)) \subseteq c l\left(f^{-1}(B), r\right)$ for $B \in I^{Y}$. 


\section{References}

[1] S. E. Abbas, "Fuzzy beta-irresolute functions", Applied Mathematics and Computation, vol. 157, pp. 369-380, 2004.

[2] C. L. Chang, "Fuzzy topological spaces", J. Math. Anal. Appl., vol. 24, pp. 182-190, 1968.

[3] K. C. Chattopadhyay, R. N. Hazra, and S. K. Samanta, "Gradation of openness : Fuzzy topology", Fuzzy Sets and Systems, vol. 49, pp. 237-242, 1992.

[4] S. J. Lee and E. P. Lee, "Fuzzy $r$-preopen and fuzzy $r$-precontinuous maps", Bull. Korean Math. Soc., vol. 36, pp. 91-108, 1999.

[5] S. J. Lee and E. P. Lee, "Fuzzy $r$-continuous and fuzzy $r$-semicontinuous maps", Int. J. Math. Math. Sci., vol. 27, pp. 53-63, 2001.

[6] S. J. Lee and E. P. Lee, "Fuzzy $r$-regular open sets and fuzzy almost $r$-continuous maps", Bull. Korean Math. Soc., vol. 39, pp. 91-108, 2002.
[7] W. K. Min and M. H. Kim, "Fuzzy $r$-minimal semiopen sets and fuzzy r-M semicontinuous functions on fuzzy r-minimal spaces", Proceedings of KIIS Spring Conference 2009, vol. 19, no. 1, pp. 4952, 2009.

[8] A. A. Ramadan, 'Smooth topological spaces", Fuzzy Sets and Systems, vol. 48, pp. 371-375, 1992.

[9] Y. H. Yoo, W. K. Min and J. I. Kim. "Fuzzy rMinimal Structures and Fuzzy $r$-Minimal Spaces", Far East Journal of Mathematical Sciences, vol. 33, no. 2, pp. 193-205, 2009.

[10] L. A. Zadeh, "Fuzzy sets", Information and Control, vol. 8, pp. 338-353, 1965.

\section{Won Keun Min}

Professor of Kangwon National University Research Area: Fuzzy topology, General topology E-mail : wkmin@kangwon.ac.kr 